\title{
Absolute response of Fuji imaging plate detectors to picosecond-electron bunches
}

\author{
K. Zeil, ${ }^{1, a)}$ S. D. Kraft, ${ }^{1}$ A. Jochmann, ${ }^{1}$ F. Kroll, ${ }^{1}$ W. Jahr, ${ }^{1}$ U. Schramm, ${ }^{1}$ L. Karsch, ${ }^{2}$ \\ J. Pawelke, ${ }^{2}$ B. Hidding, ${ }^{3}$ and G. Pretzler ${ }^{3}$ \\ ${ }_{1}^{1}$ Forschungszentrum Dresden-Rossendorf (FZD), Dresden 01314, Germany \\ ${ }^{2}$ TU Dresden, OncoRay-Radiation Research in Oncology, Dresden 01307, Germany \\ ${ }^{3}$ Heinrich-Heine Universität Düsseldorf, Düsseldorf 40225, Germany
}

(Received 2 October 2009; accepted 14 December 2009; published online 26 January 2010)

\begin{abstract}
The characterization of the absolute number of electrons generated by laser wakefield acceleration often relies on absolutely calibrated FUJI imaging plates (IP), although their validity in the regime of extreme peak currents is untested. Here, we present an extensive study on the dependence of the sensitivity of BAS-SR and BAS-MS IP to picosecond electron bunches of varying charge of up to $60 \mathrm{pC}$, performed at the electron accelerator ELBE, making use of about three orders of magnitude of higher peak intensity than in prior studies. We demonstrate that the response of the IPs shows no saturation effect and that the BAS-SR IP sensitivity of 0.0081 photostimulated luminescence per electron number confirms surprisingly well data from previous works. However, the use of the identical readout system and handling procedures turned out to be crucial and, if unnoticed, may be an important error source. (C) 2010 American Institute of Physics. [doi:10.1063/1.3284524]
\end{abstract}

\section{INTRODUCTION}

In the field of relativistic laser plasma physics, where intense pulses of electrons can be accelerated to energies of up to $1 \mathrm{GeV}$ in "table top" class experiments ${ }^{1}$ the use of compact and robust magnetic spectrometers has become the backbone for the study of the acceleration processes. Major beam characteristics such as energy spectra and beam profiles are commonly recorded online by means of phosphor screens such as Lanex. Yet, the determination of the absolute bunch charge especially for high-energy electrons, ${ }^{2,3}$ and also for protons, ${ }^{4}$ often relies on the photoluminescence detector system imaging plate (IP) by FUJI photo film (Japan) directly or indirectly, when applied for the calibration of Lanex measurements. The main reason for that are difficulties associated with the use of online measurement devices due to the massive electromagnetic noise (EMP), omnipresent in the laser-matter interaction. EMP tends to spoil electronic reference measurements with Faraday cups (FCs), ionization chambers (ICs), and also integrated current transformers (ICTs). As a nonelectronic readout system, IPs not only are immune against EMP, but moreover provide extremely high sensitivity, linear response over a large dynamic range, ${ }^{5}$ and high spatial resolution. Together with the FUJI scanner hardware and integrated software they present an easily manageable device with high stability and reproducibility. In literature information on correct handling, temperature dependence, ${ }^{6}$ fading influence, ${ }^{7}$ and absolute charge sensitivity as function of electron energy are readily available. ${ }^{8-10}$ The latter was performed with electrons that exhibit a nanosecond pulse structure. However, as laseraccelerated electron (or other particle) bunch durations are

${ }^{a)}$ Electronic mail: k.zeil@fzd.de. expected to be below 40 fs (Ref. 11) and bunch charges may reach up to several $100 \mathrm{pC}$, the linear response of these detectors (as well as of other electronic systems) at such high peak currents has been heavily discussed. Aside from experimental readout this refers to radiation protection issues and especially future medical applications as well.

Recently, we therefore developed and established an irradiation site at the electron accelerator ELBE (Refs. 12 and 13) where online and offline detector devices can be tested and calibrated. ELBE provides electron energies of up to 40 $\mathrm{MeV}$ and bunch charges of up to $77 \mathrm{pC}$. In combination with the short pulse duration of about $2 \mathrm{ps}$ and the option to choose pulse trains of variable length or single pulses with tunable charge, ELBE allows for a good simulation of laser wakefield acceleration conditions. In order to study saturation effects of the IPs as well as to collect precise calibration data with single electron bunches the ELBE peak current has been optimized and the bunch charge precisely measured with multiple charge monitors (FC and ICT). With the help of the independently measured data, a direct cross calibration of detector systems commonly used in laser plasma physics such as Lanex ${ }^{14}$ and also of EMP sensitive dosimeters as ICs or diamond detectors ${ }^{15}$ can be performed.

In the presented work, we want to focus on the absolute calibration of the response of FUJI IP types BAS-SR and BAS-MS as function of the electron bunch charge at high peak intensities. The following Sec. II gives a detailed characterization of the IPs, including handling and readout procedures. Key parameters such as the influence of fading and different scanner types (BAS5000 and BAS1800II) on the results are investigated. In the last section, Sec. III, the dependence of the IP signal on different electron bunch charges is analyzed and their sensitivity determined. 


\section{CHARACTERIZATION OF THE IP SYSTEMS}

IPs are photostimulable phosphor screens $\left[\mathrm{BaFBr}: \mathrm{Eu}^{2+}\right]$ supported by flexible metal substrates, an overview of different types being given in Ref. 16. Ionizing radiation excites electron-hole pairs in the sensitive phosphor layer. Although some of those electron-hole pairs directly recombine and scintillate, others are trapped and can later be detected through photostimulated luminescence (PSL). This can be accomplished with commercially available scanner systems such as FUJI BAS-1800II and BAS-5000, which were used in the present study.

In this paper we concentrate on two types of IPs: BAS-SR and BAS-MS provided by FUJI. They are commonly used in laser plasma experiments and easily manageable due to their protective layer. Under the influence of ambient light and temperature the IP response can be significantly reduced. ${ }^{6}$ Therefore, special care has to be taken not to expose them to light and to keep them under stable temperature conditions $\left(21^{\circ} \mathrm{C}\right)$.

\section{A. IP calibration with $\mathrm{x}$ rays}

For the calibration measurements, the IPs were cut into small pieces of $5 \times 5 \mathrm{~cm}^{2}$ and mounted on a support slab during readout scanning. All the data reported in this study were obtained using the same scanning parameters: resolution $200 \mu \mathrm{m}$, latitude parameter 5, and sensitivity parameter 4000. A 16 bit image data were recorded. Additionally, for both scanning systems the scanning region, previously verified to be sufficiently homogeneous, was kept constant. After scanning, the obtained raw image data (gray values) were directly converted into the FUJI PSL format that assures linear response.

In a first series of experiments a heavily filtered (3.54 $\mathrm{mm} \mathrm{Pb}$ and $7 \mathrm{~mm} \mathrm{Be}$ inherent filtering) high voltage $\mathrm{x}$-ray tube with tungsten anode (Isovolt 320 HS provided by Pantak Seifert) was used as reference irradiation source. In combination with a calibrated IC-based dosimeter system, stable temporal and spatial irradiation conditions could be provided. The relative dose uncertainty of the system amounts to $<3 \%$. $^{17}$ The calibrated radiation field of a x-ray tube allows for an easy provision of a large amount of irradiated reference samples as well as the opportunity to transfer absolute calibration data. For reference irradiation, the samples were exposed to $100 \mu \mathrm{Gy}$ using $200 \mathrm{kV}$ accelerating voltage. By applying multiple scans the dynamic range of the IP readout can be extended although the scanner saturates. ${ }^{16}$ Using the reference samples described above it was possible to rescale the image data after the multiple scans.

\section{B. Fading}

Due to a relaxation of the excited electron-hole pairs, the signal decreases when the time delay between illumination and readout is increased. Figure 1 shows the data points obtained in a measurement for both IP types. For further analysis the data points collected here were fitted according to empirical functions with exponential decay including two time constants

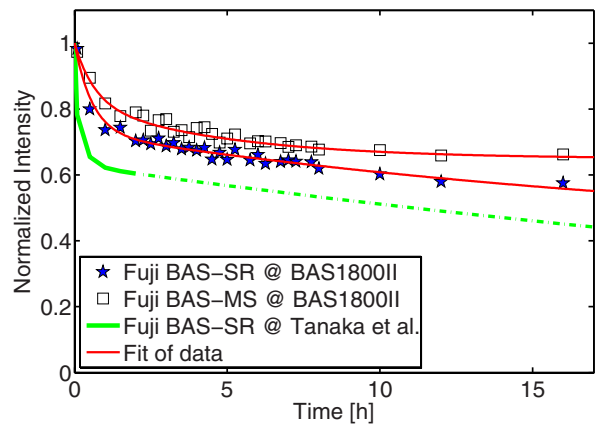

FIG. 1. (Color online) Comparison of the measured fading behavior for BAS-MS and BAS-SR IPs with published data [solid line (Ref. 8), dashed line extrapolation of Ref. 8 to longer times]. The depicted data and the fitted functions are normalized at $t=0$.

$$
\begin{aligned}
f_{\mathrm{MS}}(t)= & 6279.5+1731.2 \times \exp (-t / 0.6)+1665.5 \\
& \times \exp (-t / 4.8), \\
f_{\mathrm{SR}}(t)= & 914.2+627.5 \times \exp (-t / 0.6)+762.3 \\
& \times \exp (-t / 22.3) .
\end{aligned}
$$

Due to practical reasons the fading measurements reported here were not extended to very short times below 4 min after exposure. For this reason, in the empirical functions [Eq. (1)] no short relaxation time constant is included, leading, when extrapolated to $t=0$, to less signal compared to literature. ${ }^{8}$ In the time range of $20 \mathrm{~min}$ to $4 \mathrm{~h}$ readout after exposure, typical for laser plasma experiments, the curves show a similar behavior. However, the obtained fading coefficients $f_{\mathrm{SR}}(t)$ differ by about 10\%-20\% from literature. For $9 \mathrm{~h}$ after irradiation, which is about the time between irradiation and readout applied in this study, the exact value to be considered amounts to $18 \%$.

\section{Comparison of scanner systems}

Apart from the response of a particular IP sample the response of the specific scanner system has to be taken into account as well. For the estimation of scanner influences we measured the IP response as a function of the dose using the two readout systems BAS-1800II and BAS-5000. Therefore one set of IPs for each type was prepared and irradiated with different doses from 0.05 to $1 \mathrm{mGy}$. The readout was performed with the two different scanners for each set. During the whole procedure BAS-SR and BAS-MS type IPs were treated identically to avoid ambiguities. After scanning fading was corrected for according to Eq. (1).

The IP response corresponding to the different types of IPs and readout systems is plotted in Fig. 2. Although each individual data set shows nice linearity, obviously, the scanner responses can differ dramatically depending on the IP type. According to the manufacturer this can be explained by differences in the optical system of the scanner device. The confocal configuration of the BAS5000 allows for a better light collection than the light collecting guide of the BAS1800II in combination with the BAS-SR IP. Due to their higher sensitivity this effect is less pronounced in the case of BAS-MS type IPs. However, precise calibration factors can be determined to take into account the scanner influence. 


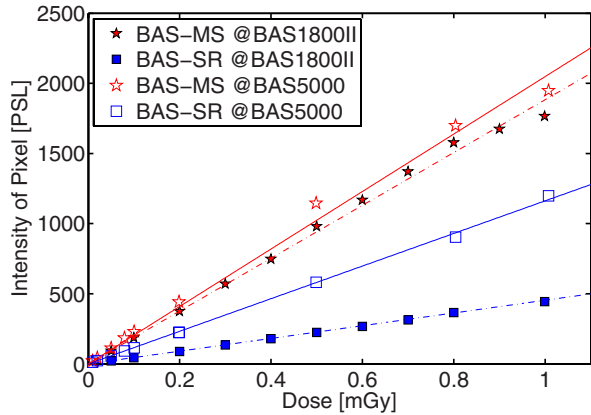

FIG. 2. (Color online) Comparison of the effective IP response when different scanning systems are used.

From the linear fits of the IP response as function of the dose $f(D)=a_{\text {scanner }}^{\text {IP-tye }} \times D$ for each data set, the sensitivity parameters $a_{\text {scanner }}^{\text {IP-type }}$ are listed in Table I. Thus the ratios

$$
\begin{aligned}
& a_{\mathrm{BAS} 5000}^{\mathrm{SR}} / a_{\mathrm{BAS} 1800 \mathrm{II}}^{\mathrm{SR}}=2.56 \pm 0.04, \\
& a_{\mathrm{BAS} 5000}^{\mathrm{MS}} / a_{\mathrm{BAS} 18001 \mathrm{II}}^{\mathrm{MS}}=1.07 \pm 0.05
\end{aligned}
$$

have to be taken into account when comparing data at different sites.

\section{IP BUNCH CHARGE DEPENDENCE}

\section{A. Experimental procedure}

The experiments were performed in the radiation physics cave at ELBE. ${ }^{18}$ The specific setup used for the irradiation is presented in Fig. 3. The electron beam exits the vacuum beam line through a beryllium window $(100 \mu \mathrm{m}$ thick, 19 $\mathrm{mm}$ diameter) and a collimator is used to shield the IPs from background radiation. The electron beam size can be tuned by several beam line elements to minimize the focal spot and maximize the electron yield at the irradiation position. In order to measure the bunch charge two independent monitors were permanently implemented. A Bergoz ICT (ICT-050070-5:1) (ICT1) and a FC (Ref. 15) both with in-house calibrated readout electronics. These devices were cross calibrated with the ELBE electron beam. Additionally an integrated system of a less sensitive Bergoz ICT (ICT-122070-10:1) (ICT2) in combination with a Bergoz boxcar integrator (BCM) was used to benchmark our charge monitors for high bunch charges. Figure 4 shows the detector responses where the FC and ICT2 signal is plotted relative to the ICT1 signal, the latter being indicated by the horizontal line. In the bunch charge range used, all charge monitors are in good agreement and a systematic charge uncertainty of $<5 \%$ can be estimated. During the measurement only ICT1 was used as nonintrusive charge reference. The fast low

TABLE I. Overview of the linear fitting parameter $a_{\text {scanner }}^{\text {IP-tye }}$ for the curves in Fig. 2 according to IP type and scanner system.

\begin{tabular}{lcc}
\hline \hline & \multicolumn{2}{c}{$\begin{array}{c}a \\
\text { (PSL/mGy) }\end{array}$} \\
\cline { 2 - 3 } \multicolumn{1}{c}{ Readout system } & BAS-SR & BAS-MS \\
\hline BAS 5000 & $1163 \pm 18$ & $2027 \pm 96$ \\
BAS 1800II & $454 \pm 3$ & $1890 \pm 31$ \\
\hline \hline
\end{tabular}

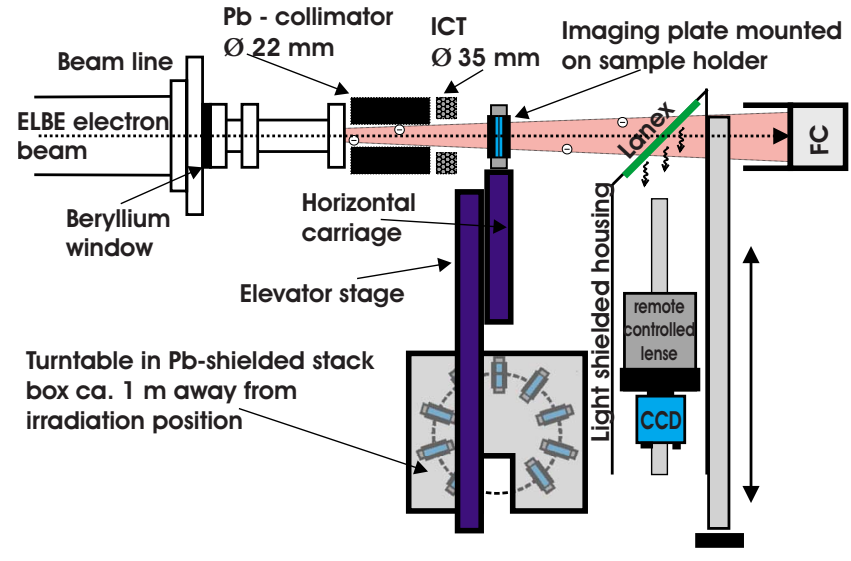

FIG. 3. (Color online) Schematic top view of the experimental setup in the radiation physics cave at ELBE showing the setup of Lanex screen (beam profile), FC (charge), and RCIS for sample handling.

noise high frequency amplifiers of ICT1 allow for the resolution of multiple pulses in pulse trains (see bottom Fig. 4). The signal can be integrated to deduce the charge of a whole pulse train.

In order to optimize the beam focus we used a phosphorescence screen (Lanex) signal, digitized by a chargecoupled device installed behind the irradiation position. At minimum a beam diameter of about $7 \mathrm{~mm}(2 \sigma$ of Gaussian beam shape) could be reached by tuning the beam focusing elements of the beam line.

Using the recently implemented single pulse mode ELBE can deliver single bunches with varying charge of up to $77 \mathrm{pC}$ as well as trains of pulses with a pulse repetition rate of $13 \mathrm{MHz}$. In this study the accessible range of bunch charges was limited to $0.3-60 \mathrm{pC}$ for a single pulse, where the lower limit is due to the resolution of the charge monitors. With pulse trains the upper limit can in general be extended by taking into account saturation effects and integration time of the detectors under investigation while, in this study, only single and double pulses were used.

Finally, different online detectors or dosimeters ${ }^{15}$ can be introduced and positioned using a linear translation stage.
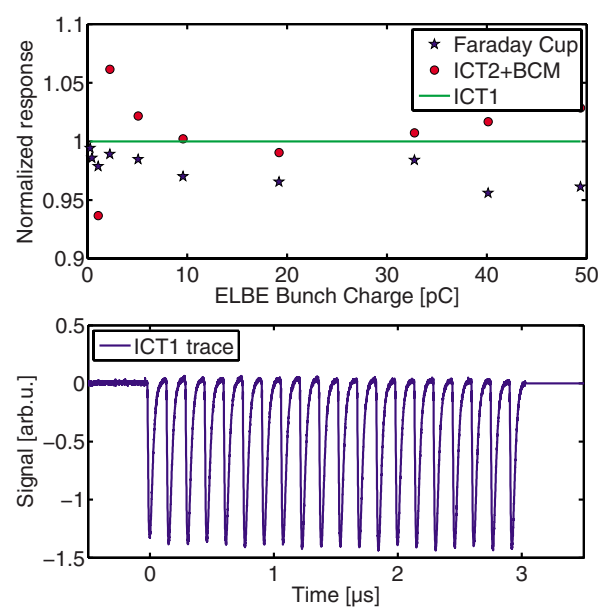

FIG. 4. (Color online) Top: Comparison of the responses of the charge monitors used. Signals from FC and ICT2 are normalized to ICT1 (horizontal line). Bottom: Typical ICT1 signal of a pulse train of 20 bunches resolving individual bunches. 

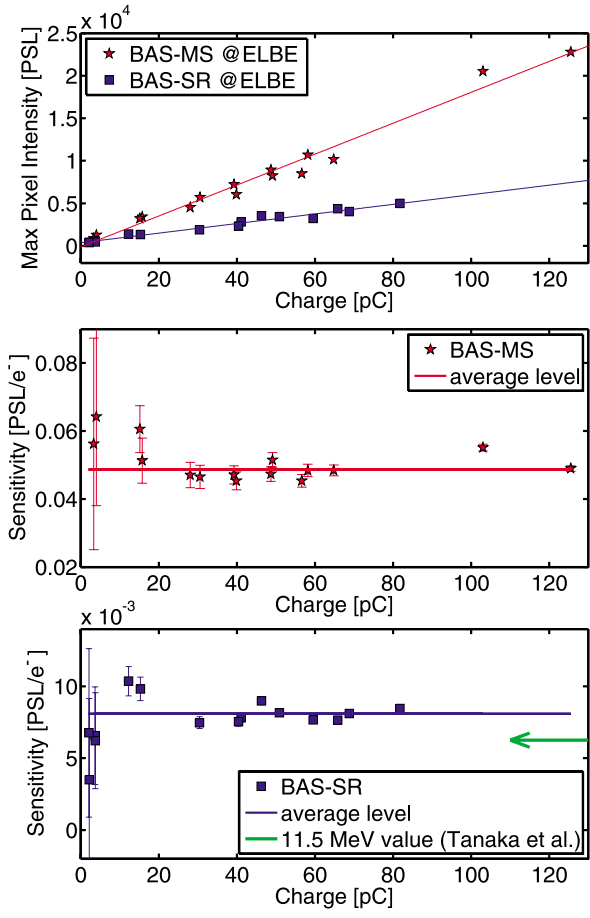

FIG. 5. (Color online) Top: PSL values of the maximum pixel as function of total bunch charge. Beneath: Sensitivity of BAS-MS and BAS-SR to number of electrons as function of the applied bunch charge and comparison with the best corresponding value for electrons of $11.5 \mathrm{MeV}$ energy measured in Ref. 8. For better comparison with literature the sensitivity values have been adapted for the BAS1800II readout system with the coefficients derived above (see Table I) and the difference in the fading curves was considered.

Due to special radiation protection regulations considering activation of air and beam line components, closing and opening of the radiation protection cave is a very time consuming procedure. Thus, irradiations of a large amount of offline detectors within one experimental run can only be realized by the use of a completely remote controlled irradiation system (RCIS, see Fig. 3). The RCIS consists of a turntable in a stack box for up to 27 samples, each of which can be separately transported into the beam position and back by linear stages (see Ref. 17). Inside the lead-shielded stack box, remaining samples are well protected against background radiation.

For irradiation at ELBE samples of both FUJI IP types BAS-SR and BAS-MS were prepared. The samples were covered by aluminum foil (13 $\mu \mathrm{m}$ thick) for protection against ambient light. Two samples were mounted together with the sensitive layers facing each other to guarantee high reproducibility during the irradiation and analysis as both layers are assumed to have been exposed to the same dose at the same energy. In two experimental runs 30 sets consisting of two IP samples each were exposed to a single electron pulse with an electron energy of $20 \mathrm{MeV}$ and different bunch charge. For charges of more than $60 \mathrm{pC}$ two consecutive bunches were applied. The charge was obtained by integration of the ICT1 signal, which was recorded for every single shot. Additionally, for both runs, several IP samples were kept in the stack box of the RCIS for background estimation.

After about $12 \mathrm{~h}$ the IP samples were read out with the FUJI BAS 5000 scanner system. Special care was taken not
TABLE II. Overview of the obtained sensitivity values $S$ depending on type of FUJI IP and scanner system. Errors are calculated with the weighted average function.

\begin{tabular}{lcc}
\hline \hline Scanner & $S_{\mathrm{SR}}\left(\mathrm{PSL} / \# \mathrm{e}^{-}\right)$ & $S_{\mathrm{MS}}\left(\mathrm{PSL} / \# \mathrm{e}^{-}\right)$ \\
\hline BAS 5000 & $0.0207 \pm 0.0002$ & $0.0534 \pm 0.0005$ \\
BAS 1800II & $0.00809 \pm 0.00007$ & $0.0499 \pm 0.0005$ \\
\hline \hline
\end{tabular}

to expose the IPs to ambient light. During each scanning sequence a BAS-SR and BAS-MS reference sample (0.1 mGy by $200 \mathrm{kV} x$ rays) was introduced into the scanner as well. This ensured correlation and rescaling of the data obtained from multiple scans and the deduction of the error evolving from the scanning procedure. Multiple scans were necessary whenever the readout of an IP sample saturated. The obtained image data were directly converted into the PSL format and the PSL values were corrected according to the fading functions [see Eq. (1)].

The beam profile was analyzed via a two-dimensional Gaussian fitting function

$$
g(x, y)=g_{0}+A \exp \left[-\left(x-x_{c}\right)^{2} / 2 \sigma_{x}^{2}-\left(y-y_{c}\right)^{2} / 2 \sigma_{y}^{2}\right],
$$

where $g_{0}$ is the offset, $A$ the amplitude parameter, and $x_{c}, y_{c}$ and $\sigma_{x}, \sigma_{y}$ describe the center and width in $x$ and $y$ directions, respectively. Likewise, mean PSL values from the background $B G$ as well as reference samples $\mathrm{REF}_{i}$ for each scanning sequence $i$ were extracted. Background radiation, mainly originating from bremsstrahlung produced when electrons are lost in beam line elements or beam dumps, has a minor influence on the measurement. That can be seen by the fraction of $B G / A$ which was less than 0.005 . This value is in good agreement with the fitting offset parameter $g_{0}$ for which a ratio between $g_{0}$ and $A$ of less than 0.001 was obtained, considering all samples. With the help of the $\mathrm{REF}_{i}$ values the PSL values for IP samples which were scanned twice or even three times were rescaled.

\section{B. IP sensibility}

The obtained amplitude values $A$ for all samples are plotted in the top of Fig. 5. Evidently, the response of the FUJI IP shows linear behavior and no saturation effect is visible. In order to obtain the exact response of the IPs to a certain electron number a special feature of the electron accelerator has to be taken into account. Although the electron gun is electronically switched off, there is a very small leakage current running through the entire beam line. Especially for the irradiation of IPs as offline detectors with single pulses of low charge, this leakage leads to a considerable amount of collected charge, depending on the rest time of the samples in the irradiation position, which was adjusted to be constant. To cope with this peculiarity the following procedure was applied. First, the integral $\int g(x, y) d x d y=2 \pi \sigma_{x} \sigma_{y} A$ over the entire beam spot for each sample is calculated. Subsequently, the linear dependence between IP response and bunch charge was fitted with $f(Q)=a \times Q+n$. By treating $n$ as fitting parameter the leakage current was determined as an offset for all IP responses and hence subtracted. Finally, the IP sensitivity as fraction of integrated PSL signal to electron 
TABLE III. Overview of beam parameters used in previous studies (Refs. 8 and 10): electron energy $E$, bunch duration $\tau$, focal spot, peak intensity, and measured IP responses (BAS-SR). Comparison with the data obtained at ELBE.

\begin{tabular}{lcrcccc}
\hline \hline LINAC & $\begin{array}{c}E \\
(\mathrm{MeV})\end{array}$ & $\begin{array}{c}Q \\
(\mathrm{pC})\end{array}$ & $\begin{array}{c}\tau \\
(\mathrm{ps})\end{array}$ & $\begin{array}{c}\text { Focal spot } \\
\left(\mathrm{cm}^{2}\right)\end{array}$ & $\begin{array}{c}\text { Intensity } \\
\left(\# \mathrm{e}^{-} \mathrm{ps}^{-1} \mathrm{~cm}^{-2}\right)\end{array}$ & $\begin{array}{c}\text { IP response } \\
\left(\mathrm{PSL} / \# \mathrm{e}^{-}\right)\end{array}$ \\
\hline L-Band & 11.5 & 50 & 5000 & $\approx 7$ & $8.9 \times 10^{3}$ & 0.0074 \\
L-Band & 30 & 50 & 5000 & $\approx 7$ & $8.9 \times 10^{3}$ & 0.007 \\
S-Band & 100 & 2500 & 5000 & $\approx 28$ & $1.1 \times 10^{5}$ & 0.0064 \\
SPring-8 & 1000 & 10 & 1000 & $\approx 1$ & $6.2 \times 10^{4}$ & 0.0041 \\
ELBE & 20 & 60 & 2 & 0.4 & $4.68 \times 10^{8}$ & 0.0081 \\
\hline \hline
\end{tabular}

number was calculated and is plotted as a function of the bunch charge in Fig. 5. The errors in the pictures, mostly due to the different rest time of the samples in the irradiation position, are derived from the fitting parameter $n$. As can be seen the sensitivity of the IPs remains constant for the investigated bunch charge range. By means of a weighted average an overall sensitivity of the different IP types is calculated (see Table II) and compared to published data for the case of the BAS-SR type IPs (see bottom of Fig. 5). The obtained sensitivity is in good agreement with values reported in literature, provided the same readout components are used or the corrections, listed in Eq. (2) are made. According to the discussion in Sec. II B and for better comparison, the sensitivity from Ref. 8 is divided by a factor of 1.18 to take into account the different fading curves.

In order to estimate the absolute uncertainty of the presented calibration measurement beyond the statistical errors depicted in Table II, an extensive error analysis has been performed. As already mentioned the relative error of the bunch charge measurement amounts to 5\% (Fig. 4). The influence of background radiation has been found to be negligible and errors resulting from the fitting of the beam profiles, e.g., the errors of the fitting parameters amount to $<1 \%$. From the analysis of the reference samples present during the scanning sequences fluctuations of $<2 \%$ could be deduced.

\section{CONCLUSION}

We present an extensive study on the bunch charge dependence of the response of BAS-SR and BAS-MS FUJI IPs at the electron accelerator ELBE. Using a x-ray tube an unambiguous reference has been established. No saturation effects have been detected in the charge range of interest, although the peak intensity exceeds values from previous studies by about three orders of magnitude (see Table III). This has been achieved by the use of a picosecond pulse structure and a smaller spot size than applied in Refs. 8 and 10. The calibration data reported therein, which are commonly used in laser plasma experiments, are reproduced with a small systematic difference of about $28 \%$. The FUJI IP system shows amazingly high linearity and reproducibility when and only when identical readout components are used. Otherwise systematic errors of more than $100 \%$ are possible.

\section{ACKNOWLEDGMENTS}

We are very grateful for discussions with the ELBE crew and for their support of this work. Colleagues from the $\mathrm{Mu}-$ nich Center of Advanced Photonics are acknowledged for the loan of one ICT as well as the BCM. This work was supported by DFG TR 18 project. This work has been partially supported by LASERLAB-EUROPE/LAPTECH, EC FP7 Contract No. 228334.

${ }^{1}$ W. Leemans, B. Nagler, A. Gonsalves, C. Toth, K. Nakamura, C. Geddes, E. Esarey, C. Schroeder, and S. Hooker, Nat. Phys. 2, 696 (2006).

${ }^{2}$ Y. Glinec, J. Faure, A. Guemnie-Tafo, V. Malka, H. Monard, J. Larbre, V. De Waele, J. Marignier, and M. Mostafavi, Rev. Sci. Instrum. 77, 103301 (2006).

${ }^{3}$ S. Karsch, J. Osterhoff, A. Popp, T. Rowlands-Rees, Z. Major, M. Fuchs, B. Marx, R. Hörlein, K. Schmid, L. Veisz, S. Becker, U. Schramm, B. Hidding, G. Pretzler, D. Habs, F. Grüner, F. Krausz, and S. M. Hooker, New J. Phys. 9, 415 (2007).

${ }^{4}$ A. Mančić, J. Fuchs, P. Antici, S. Gaillard, and P. Audebert, Rev. Sci. Instrum. 79, 073301 (2008).

${ }^{5}$ S. Gales and C. Bentley, Rev. Sci. Instrum. 75, 4001 (2004).

${ }^{6} \mathrm{H}$. Ohuchi and A. Yamadera, CYRIC Annual Report 2001.

${ }^{7}$ H. Ohuchi, A. Yamadera, and T. Nakamura, Nucl. Instrum. Methods Phys. Res. A 450, 343 (2000).

${ }^{8}$ K. Tanaka, T. Yabuuchi, T. Sato, R. Kodama, Y. Kitagawa, T. Takahashi, T. Ikeda, Y. Honda, and S. Okuda, Rev. Sci. Instrum. 76, 013507 (2005).

${ }^{9}$ H. Chen, N. Back, T. Bartal, F. Beg, D. Eder, A. Link, A. MacPhee, Y. Ping, P. Song, A. Throop, and L. Van Woerkom, Rev. Sci. Instrum. 79, 033301 (2008).

${ }^{10}$ N. Nakanii, K. Kondo, T. Yabuuchi, K. Tsuji, K. Tanaka, S. Suzuki, T. Asaka, K. Yanagida, H. Hanaki, T. Kobayashi, K. Makino, T. Yamane, S. Miyamoto, and K. Horikawa, Rev. Sci. Instrum. 79, 066102 (2008).

${ }^{11}$ A. D. Debus, M. Bussmann, U. Schramm, R. Sauerbrey, C. D. Murphy, Zs. Major, R. Hörlein, L. Veisz, K. Schmid, J. Schreiber, K. Witte, S. P. Jamison, J. G. Gallacher, D. A. Jaroszynski, M. C. Kaluza, B. Hidding, S. Kiselev, R. Heathcote, P. S. Foster, D. Neely, E. J. Divall, C. J. Hooker, J. M. Smith, K. Ertel, A. J. Langley, P. Norreys, J. L. Collier, and S. Karsch, "Electron bunch length measurements from laser-accelerated electrons using single-shot THz time-domain interferometry," Phys. Rev. Lett. (submitted).

${ }^{12}$ F. Gabriel, P. Gippner, E. Grosse, D. Janssen, P. Michel, H. Prade, A. Schamlott, W. Seidel, A. Wolf, and R. Wünsch, Nucl. Instrum. Methods Phys. Res. B 161, 1143 (2000).

${ }^{13}$ www.fzd.de/db/Cms?pNid=145.

${ }^{14}$ A. Buck, K. Zeil, A. Popp, K. Schmid, A. Jochmann, S. Kraft, B. Hidding, T. Kudyakov, C. Sears, L. Veisz, S. Karsch, J. Pawelke, R. Sauerbrey, T. Cowan, F. Krausz, and U. Schramm, "Absolute charge calibration of scintillating screens for relativistic electron detection," Rev. Sci. Instrum. (unpublished).

${ }^{15}$ L. Karsch, E. Bayreuther, S. Kraft, C. Richter, U. Schramm, K. Zeil, and J. Pawelke, "Dose rate dependence for different dosimeters and detectors" (unpublished). 
${ }^{16}$ B. Hidding, G. Pretzler, M. Clever, F. Brandl, F. Zamponi, A. Lübcke, T. Kämpfer, I. Uschmann, E. Förster, U. Schramm, R. Sauerbrey, E. Kroupp, L. Veisz, K. Schmid, S. Benavides, and S. Karsch, Rev. Sci. Instrum. 78, 083301 (2007).
${ }^{17}$ K. Zeil, E. Beyreuther, E. Lessmann, W. Wagner, and J. Pawelke, Nucl. Instrum. Methods Phys. Res. B 267, 2403 (2009).

${ }^{18}$ W. Neubert, B. Azadegan, W. Enghardt, K. Heidel, J. Pawelke, and W. Wagner, Nucl. Instrum. Methods Phys. Res. B 254, 319 (2007). 